A. Weisbecker, M. Burmester \& A. Schmidt (Hrsg.): Mensch und Computer 2015 Workshopband, Stuttgart: Oldenbourg Wissenschaftsverlag, 2015, S. 391-397.

\title{
A Survey: Outdoor Mobility Experiences by the Visually Impaired
}

\author{
Limin Zeng
}

Human-Computer Interaction Group, TU Dresden

\begin{abstract}
This paper presents an ongoing survey with 97 blind and visually impaired people. The survey investigates their outdoor travel experience and mobility related barriers, and usages of mobile devices and GPS navigation tools. These findings might help developers and researchers to understand the barriers suffered by blind and visually impaired pedestrians, and find solutions to resolve the reported issues, such as GPS navigation systems lacking of environmental accessibility data.
\end{abstract}

\section{Introduction}

As one important part of daily life activities, outdoor journey is significant for people to participate in various social activities, such as going to office or shopping. In general, disabled citizens practically expect special requirements offered by mobility aids. For instance, while using navigation systems wheelchair users prefer a route without high steps and the blind might expect a quiet and non-obstacle sidewalk rather than the shortest one.

For blind and visually impaired individuals, their mobility-related requirements have been changed as the development of information and communication technology (ICT) in recent years. On one hand, there are more and more barrier-free public facilities within cities. On the other hand, nowadays, a large number of people with visual impairments make use of mobile devices and related services, such as GPS-based navigation services on dedicated products (e.g. Kapten ${ }^{1}$, BrailleNotes GPS ${ }^{2}$ and Trekker Breeze ${ }^{3}$ ), or on smart phones (e.g. Loadstone $\mathrm{GPS}^{4}$ ) to navigate to their destinations. However, their experiences and sufferings while travelling in urban environments and their experiences with assistive systems are unclear.

\footnotetext{
1 Kapten GPS, http://www.kapsys.com/

2 BrailleNotes GPS, http://www.senderogroup.com

3 Trekker Breeze, http://www.humanware.com

4 Loadstone-GPS, http://www.loadstone-gps.com/
} 
In contrast to mobility impaired pedestrians who suffer various physical environmental barriers while travelling outdoor, such as slopes, and curbs (Meyers et al, 2002; Beale et al, 2006), blind and visually impaired pedestrians face a series of barriers in daily life, from accessing Internet (Bayer \& Pappas, 2006; van Puffelen et al., 2008), to various mobilityrelated barriers like walking on ascending/descending stairs (West et al., 2002) and hitting obstacles at a head level (Manduchi \& Kurniawan, 2011).

In order to discover outdoor mobility experiences of blind and visually impaired people, this paper focuses on addressing an ongoing international survey with 97 subjects. The survey consists of 2 main topics related to outdoor experiences, and usage of mobile devices while on the move. Possible solutions to improve independent and safe outdoor journeys have been discussed in the end of the paper.

\section{The Survey}

The survey was delivered to our participants in two ways, including an accessible online questionnaire and offline accessible documents distributed by special agencies, such as blind associations. An accessible web pages (in English and in Chinese) had been created and its link was advertised at several associated online forums, mail lists and communities for blind and visually impaired people. Additionally, electronic documents (in Word format) were sent to a couple of agencies, who specially provided services to the visually impaired. Those agencies were in charge of distributing the questionnaire to our participants, and returning all responses they received finally. Apart from investigating outdoor mobility experiences, the survey would consider to find differences between developing countries (e.g., China, India) and developed countries/areas.

In addition to short free text questions (e.g., age and country) and single choice questions, the survey adopts multiple choice with comments where users are allowed to enter additional comments. The multiple choice answers mostly come from common cases or situations in daily life (Manduchi \& Kurniawan, 2011). Moreover, the multiple choice answers help participants not only better understand the corresponding questions, but also think about their own comments.

\subsection{Questions}

In addition to personal information, such as age, occupation and residence, the questions covered two main parts. Multiple choice questions are Q4, Q5, Q6, and Q9.

- Outdoor travel experience: The questions addressed users' personal experience when travelling outdoor, such as how often they travel outdoor, whether alone or with a partner, and how to pass a crossing.

Q1: Which grade do you belong to (Blind, Severe low vision, Low vision, People with sight)?

Q2: How often do you go out? 
Q3: Do you always go out alone?

Q4: When you go out, do you have those experiences?

- Usage of assistive devices and mobile phones: In this part, it is asked for assistive devices carried around to navigate, utilization of GPS devices, as well as popular mobile services.

Q5: When you go out, which devices do you often bring with you?

Q6: Excepting make a call, how do you use your mobile phone?

Q7: Do you think it is helpful with GPS navigation system when go out?

Q8: How often do you use GPS navigation system (For GPS Users only)?

Q9: What are the shortcomings of GPS navigation system, in your view (For GPS Users only)?

\subsection{Results}

- Participants

97 participants from 13 countries (e.g. China, India, UK, Germany, etc.) took part in the survey. 64 subjects $(66 \%)$ were blind and 33 subjects (34\%) were low vision. The average age was 38.3 years. The youngest subject was 19 years old while the oldest participant was 63 years old. The subjects had various occupations such as teacher, student, masseur, engineer, retired employee and unemployed persons. There were 79 responses from developing countries, and 18 ones from developed countries.

- Independent outdoor journey

Figure 1. illustrates subjects' outdoor travel frequency, from the aspects of blind subject group, low vision subject group and all subjects. $75.8 \%$ of low vision subjects and $51.6 \%$ of blind subjects travel every day, but there were $12.5 \%$ of blind subjects who had a low outdoor travel frequency (less than 1 time/week).

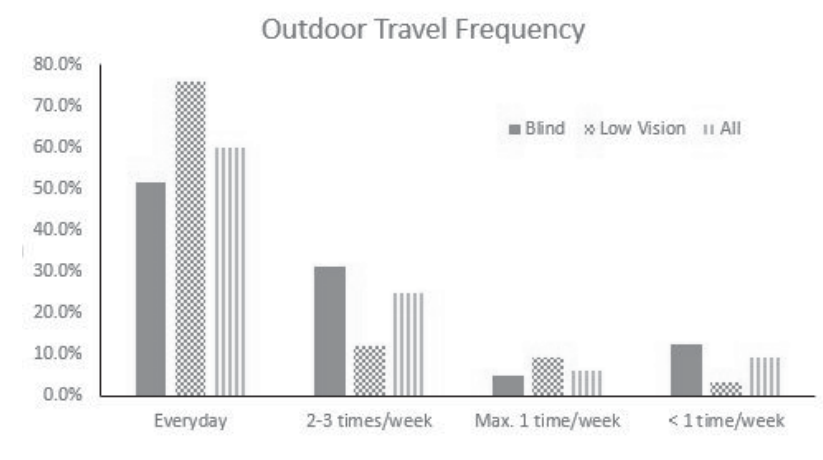

Figure 1. The subjects' outdoor travel frequency

Regarding to their independent outdoor travel, only $12.5 \%$ of blind subjects travelled alone each time, comparing with $39.4 \%$ of low vision subjects. About $50 \%$ of blind subjects travelled alone most of time, but there were still $17.2 \%$ and $18.8 \%$ of blind subjects who needed a 
partner most of time and each time, respectively. $21.2 \%$ of low vision subjects had to travel with a partner most of time or each time, see Figure 2.

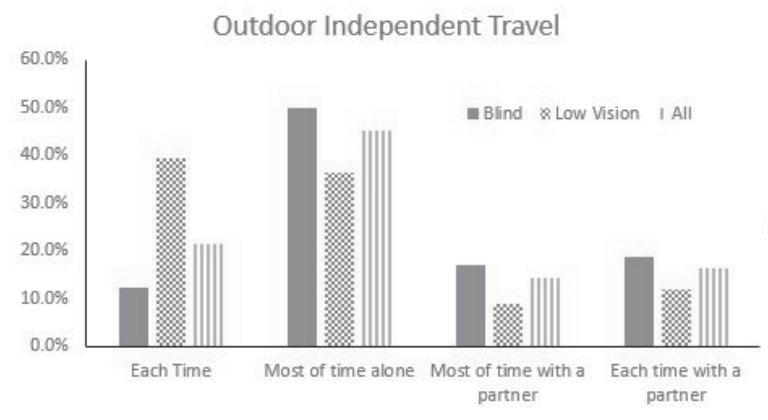

Figure 2. The subjects' independent outdoor travel

- Barriers in cities

Table 1 ranks the frequency of the 9 given barriers in Q4. Surprisingly, getting lost is not the most frequent issue. Public transport stops lacking of audio information was reported by $82.4 \%$ of participants. $81.4 \%$ of them ranked traffic lights without audio output as the second frequent barrier. In addition to audio information of facilities, various barriers, impact safety and independent of outdoor journey for people with vision impairments, such as the precise location of the entrance of a building, unknown stairs and roadside holes, as well as obstacles upon a waist level. The condition of sidewalks and crossings need to be paid more attention in the view of nearly half of the participants. Besides, there were a few additional comments made by the participants, excepting several responses which reported sidewalks for the blind are often occupied by booths and bikes.

Subjects' Suffering (\%) on the 9 Barriers

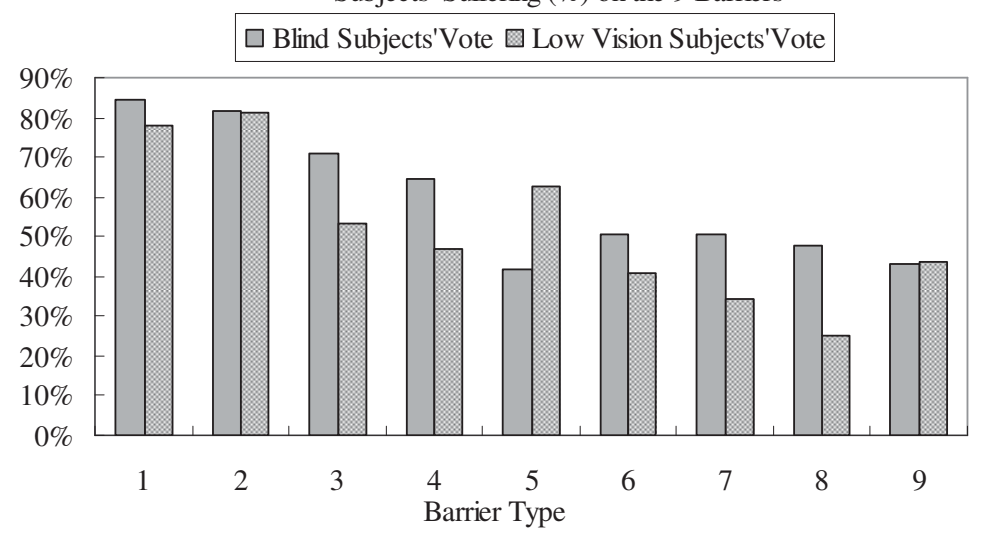




\section{Figure 3. Subjects' votes on the 9 given barriers ${ }^{5}$}

As illustrated in Figure 3, the blind subjects and the low vision subjects reported their sufferings on the 9 given barriers while travelling. Their experiences on the 9 barriers is consistent between the low vision subjects and the blind subjects (Nonparametric Chi-square $=7.03, d f=8, p=0.533$ ). Besides, the low vision subjects and the blind subjects did not have a significant difference on the suffering hazards or obstacles within Barrier 5, Barrier 6, and Barrier 8 (Nonparametric Chi-square $=5.094, d f=2, p=0.078$ ). Note that the Chi-square test adopted is at the $95 \%$ confidence level.

\begin{tabular}{clc}
\hline$\#$ & \multicolumn{1}{c}{ Barrier } & Support (\%) \\
\hline Barrier 1 & Public transport stops lack of audio information & 82.4 \\
Barrier 2 & Traffic light without audio output & 81.4 \\
Barrier 3 & Failure to find the entrance of a building & 64.9 \\
Barrier 4 & Ill-formed and irregular sidewalks & 58.7 \\
Barrier 5 & Falling down because of unknown stairs & 48.5 \\
Barrier 6 & Hitting obstacles upon a waist level & 47.4 \\
Barrier 7 & Getting lost & 45.3 \\
Barrier 8 & Falling down because of unknown roadside holes & 44.3 \\
Barrier 9 & Complex pedestrian crossings & 43.3 \\
\hline
\end{tabular}

Table 1. The rank of the frequency of the 9 given barriers

- Usage of mobile devices and services

Only two subjects did not bring a mobile phone when travelling outdoor. In other words, the acceptance of mobile phones (including smart phones) was 97.7\%. Among those mobile phone users, in addition to making a phone call, several other popular mobile services were pointed out: sending Short Message Service (70.1\%), accessing Internet (45.4\%), GPS navigation $(34.0 \%)$ and even taking photos $(30.9 \%) .95 .3 \%$ of the blind subjects used white canes, and only 3 ones walk with guide dogs. About $36 \%$ of low vision subjects were white cane users. Some subjects also brought GPS receivers (14\%), music player (57.7\%) and FM Radio $(17.5 \%)$, and 7 subjects brought a laptop for work purposes.

- GPS navigation experiences

$84.5 \%$ of subjects responded that GPS navigation tools were helpful while travelling, even including a number of subjects who were not GPS navigation users. There were 33 subjects (34\%) who had GPS navigation experiences in practical. Regarding to when they use a GPS navigation system, $42.4 \%$ (14 of 33 subjects) pointed out they used it in unknown places, and

5 The 9 Barrier Type is listed in Table 1. 
$39.4 \%$ made use of their GPS navigation system each time. Among those users, $69.7 \%$ reported the most important weak point of GPS navigation systems, is lack of environmental accessibility map data, such as whether a traffic light is with or without audio output. In Table 2, map data of GPS navigation tools (in Rank 1 and Rank 2) was more criticized than the common issues of typical GPS systems, i.e., precision and weak signals in cities.

\begin{tabular}{llc}
\hline Rank & \multicolumn{1}{c}{ Shortcoming } & Support (\%) \\
\hline 1 & Without environmental accessibility data & 69.7 \\
2 & Map data out of date & 57.6 \\
3 & Inaccuracy GPS location & 54.5 \\
4 & Weak signal of GPS in urban environment & 39.4 \\
\hline
\end{tabular}

Table 2. The rank of the frequency of the 4 given GPS Shortcomings

\subsection{Discussions}

It seems there were a considerable number of blind individuals (36\%) and low vision individuals $(21.2 \%)$ who needed sighted companions while travelling outdoor. It is important to improve their independent mobility via building accessible public facilities and developing varying assisted tools.

Due to a high acceptance of mobile phones (including smart phones) and nowadays ubiquitous wireless networks, various mobile phone based applications can be one of promising directions to improve mobility. Apart from accessible GPS navigation systems, like Ariadne GPS (2015), blind and visually impaired people would use mobile phones to detect traffic lights in the Crosswatch system (Ivanchenko et al., 2008), to answer daily questions in the VizWiz system (Bigham et al., 2010), and to detect obstacles (Tapu et al., 2013). Certainly, an accessible user interface is required for such applications.

In current GPS navigation systems, it is very helpful to contain special environmental accessibility information for blind and visually impaired individuals. Crowd-sourcing based approaches can be one of low-cost and effective methods to collect various accessible data of geographic features, like accessing information about points of interest in Blindsquare (2015) and entrances in unfamiliar areas (Zeng \& Weber, 2015).

\section{Conclusion}

This paper presents the results of our ongoing survey about blind and visually impaired people's outdoor mobility experiences. 97 subjects participated the survey. It is found that a considerable number of blind and visually impaired people did not have frequent and independent outdoor journeys. The results indicated that the current GPS navigation systems 
have problems to provide up-to-date map data and environmental accessibility data for the disabled. Considering a high acceptance of mobile phones, accessible mobile phone based applications can be one of promising directions to help blind and visually impaired people travel independently and safely. The survey will continue in developed countries, and the differences between developing countries and developed countries will be reported in the future.

\section{References}

Ariadne GPS system (2015): http://www.ariadnegps.eu/, last access: June 30th. 2015.

Bayer, N.L. \& Pappas, L., (2006). Accessibility Testing: Case history of blind testers of enterprise software. Technical Communication, Vol.53, No.1, pp.32-35.

Beale, L., Field, K. Briggs, D. Picton \& P. Matthews, H., (2006). Mapping for wheelchair users: route navigation in urban spaces. The Cartographic Journal, 2006, 43, (1), pp. 68-81

Bigham, J. P., Jayant, C., Ji, H., Little, G., Miller, A., Miller, R.C., Miller, R., Tatarowicz, A., White, B., White, S. \& Yeh, T., (2010). VizWiz: Nearly real-time answers to visual questions. In Proc. of UIST'10, ACM Press (2010), 333-342.

Blindsquare system (2015): http://blindsquare.com/, last access: June 30th. 2015.

Ivanchenko, V., Coughlan, J. \& Shen, H., (2008). Crosswatch: A camera phone system for orienting visually impaired pedestrians at traffic intersections. In Proc. of ICCHP 2008, 1122 - 1128.

Manduchi, R. \& Kurniawan, S. (2011). Mobility-related accidents experienced by people with visual impairment. Research and Practice in Visual Impairment and Blindness, 4(2), 44-54.

Meyers, A.R., Anderson, J. J., Miller, D. R., Shipp, K. \& Hoenig, H., (2002). 'Barriers, facilitators, and access for wheelchair users: substantive and methodological lessons from a pilot study of environmental effects', Social Science \& Medicine, 2002, 55, (8), pp. 1435-1446

Tapu, B., Mocanu., B., Bursuc, A. \& Zaharia, T., (2013). A smartphone-based obstacle detection and classification system for assisting visually impaired people. In Proc. of ICCVW 2013, 444 - 451.

van Puffelen, C., van der Geest, T. \& van der Meij, H., (2008). The use of digital skills by visually disabled people to participate in society. In Proc. of IADIS International Conference ICT, Society and Human Beings 2008, 85 - 90.

West, S. K., Rubin, G.S., Broman, A.T., Munoz, B., Bandeen-Roche, K. \& Turano, K. (2002). How does visual impairment affect performance on tasks of everyday life? Arch Ophthalmol, 120, 774-780.

Zeng L. \& Weber.G. (2015). A pilot study of collaborative accessibility: how blind people find an entrance. In Proc. of ACM MobileHCI 2015, 24th August -27th August, 2015, Denmark.

\section{Contact Information}

Dr. -Ing. Limin Zeng (limin.zeng@tu-dresden.de), Human-Computer Interaction Group, TU Dresden.

Address: Room 1067, Nöthnitzer str. 46, 01187 Dresden, Germany

Phone: $+49(0) 351 / 46338421$

Fax: $+49(0) 351 / 46338491$

Website: http://www.inf.tu-dresden.de/content/institutes/iai/mmc/index.en.html 
